12th International Symposium on Cosmology and

Particle Astrophysics (CosPA 2015)

International Journal of Modern Physics: Conference Series

Vol. 43 (2016) 1660193 (8 pages)

(C) The Author(s)

DOI: $10.1142 / \mathrm{S} 2010194516601939$

\title{
Axion Research at CAPP/IBS
}

\author{
SungWoo Youn \\ Center for Axion and Precision Physics Research, Institute for Basic Science (IBS) \\ Daejeon 34141 Republic of Korea \\ swyoun@ibs.re.kr
}

Published 7 July 2016

\begin{abstract}
The axion, a hypothetical fundamental particle, was postulated as an attractive solution to the $\mathrm{CP}$ problem in quantum chromodynamics and believed to be an ideal candidate for the cold dark matter. The Center for Axion and Precision Physics Research of the Institute for Basic Science has launched a state of the art experiment to search for the hypothesised new particle using microwave resonant cavities. I will discuss R\&D efforts at our center and plans for the experiment.
\end{abstract}

Keywords: Axion; Strong-CP; dark matter; CAPP/IBS.

\section{Introduction}

Charge-Parity symmetry breaking, CPV, is believe to be a major source in particle physics to the matter-antimatter imbalance in our Universe. The CPV has been well understood in the electroweak interaction with many observations consistent with predictions, e.g., CPV phase in the CKM matrix. ${ }^{1}$ However, the CPV shows a conflict between theoretical predictions and experiment measurements in the strong interaction. According to quantum chromodynamics (QCD), a CP violating term is naturally introduced to the Lagrangian due to the non-zero QCD vacuum, which predicts a sizable neutron electric dipole moment (nEDM). However, experimental limits of $\mathrm{nEDM}\left(<3 \times 10^{-26} \mathrm{e} \cdot \mathrm{cm}\right)^{2}$ are significantly smaller than the predictions $\left(\sim 10^{-17} \mathrm{e} \cdot \mathrm{cm}\right)$, which indicates QCD seems to respect the CP symmetry. Such a contradiction between theory and experiment in the QCD sector is known as the strong-CP problem. This is one of the most important but unsolved problems in physics.

In 1977, R. Peccei and H. Quinn suggested an elegant idea to solve the strongCP problem. ${ }^{3}$ They introduced a new global U(1) symmetry, called PQ symmetry, the breakdown of which invokes a pseudo-scalar field permeating all space. In this

This is an Open Access article published by World Scientific Publishing Company. It is distributed under the terms of the Creative Commons Attribution 4.0 (CC-BY) License. Further distribution of this work is permitted, provided the original work is properly cited. 
Table 1. Theoretical properties of the axion.

\begin{tabular}{lc}
\hline Interaction & Gravity, Electromagnetic \\
Mass & $10^{-6} \sim 1 \mathrm{eV} / \mathrm{c}^{2}$ \\
Electric charge & 0 \\
Spin-parity $\left(J^{P}\right)$ & $0^{-}$ \\
Local density $\left(\rho_{\text {local }}\right)$ & $0.3 \sim 0.45 \mathrm{GeV} / \mathrm{cm}^{3}$ \\
Velocity $(\beta=v / c)$ & $\sim 10^{-3}\left(\rightarrow Q_{a} \sim 10^{6}\right)$ \\
\hline
\end{tabular}

theory, similar to the Higgs mechanism where spontaneous electroweak symmetry breaking involves the Higgs boson, the PQ symmetry breaks spontaneously (and explicitly) involving a new pseudo-Goldstone boson, which was named "axion" by F. Wilczek. Discovery of the Higgs boson in 2012 has enhanced the interest in the axion and the possibility of its existence. Table 1 summaries the theoretical properties of the axion.

Furthermore, if axions exist and have low mass within a specific range (between 1 and $100 \mu \mathrm{eV}$ ), they can fit the halo model to account for the cold dark matter. ${ }^{4}$ The new particle is thus a possible component of the dark matter, as well as a solution of the strong-CP problem, and thereby it is referred to as axion dark matter.

\section{Detection Principles of Cavity-based Experiments}

Detection of the axion dark matter employs the Primakoff effect, ${ }^{5}$ where pseudoscalar particles are produced via the electromagnetic (EM) interaction of a high energy photon scattering off nuclei, e.g. $\gamma \gamma \rightarrow \pi^{0}, \rho, \ldots$ In 1983, Pierre Sikivie wrote down the modification of Maxwell's equations from a light stable axion ${ }^{6}$ and showed that axions can be detected on Earth by converting them into photons, equivalently to the inverse Primakoff effect, in EM resonators in the presence of a strong magnetic field, the principle of haloscope.

The Lagrangian of axion coupling to photons is given by

$$
L_{a \gamma \gamma}=-\frac{g_{a \gamma \gamma}}{4} \phi_{a} F_{\mu \nu} F^{\mu \nu}=-g_{a \gamma \gamma} \phi_{a} E \cdot B
$$

where $g_{a \gamma \gamma}$ is the coupling constant, $\phi_{a}$ is the axion field, and $F_{\mu \nu}$ is the EM fieldstrength tensor. $g_{a \gamma \gamma}$ is defined as $g_{a \gamma \gamma} \equiv \frac{\alpha}{\pi} \frac{g_{\gamma}}{f_{P Q}}$ with $\alpha$ being the fine structure constant, $g_{\gamma}$ being the model dependent coefficient ( 0.97 for $\mathrm{KSVZ}^{7}$ and -0.36 for $\mathrm{DFSZ}^{8}$ ), and $f_{P Q}$ being the PQ symmetry breaking scale with the following relation with the axion mass, $m_{a}$ :

$$
m_{a}=\frac{f_{\pi} m_{\pi}}{f_{P Q}} \frac{\sqrt{z}}{1+z} \approx 6 \mu e V \frac{10^{12} \mathrm{GeV}}{f_{P Q}}
$$

where $z=m_{u} / m_{d}$, the mass ratio of $u$ and $d$ quarks. 
The expected power from axion-to-photon conversion is expressed by ${ }^{6}$

$$
P_{a \gamma \gamma}=g_{a \gamma \gamma} \frac{\rho_{a}}{m_{a}} B^{2} V C \operatorname{Min}\left(Q_{L}, Q_{a}\right),
$$

where $B$ is the applied magnetic field, $V$ is the cavity volume, $C$ is the normalized form factor describing the coupling of the axion to a specific resonant mode of the cavity, $Q_{L}$ is the loaded quality factor of the cavity and $Q_{a} \approx 10^{6}$ is the quality factor of the axion signal. The experimental sensitive is described by signal-to-noise ratio $(\mathrm{SNR})$ defined as

$$
\mathrm{SNR} \equiv \frac{P_{\text {signal }}}{P_{\text {noise }}}=\frac{P_{a \gamma \gamma}}{k_{B} T_{\text {syst }}} \sqrt{\frac{t_{\text {int }}}{\Delta f_{a}}},
$$

where $k_{B}$ is the Boltzmann's constant, $T_{\text {syst }}$ is the system noise temperature, $t_{\text {int }}$ is the integration time, $\Delta f_{a}$ is the signal bandwidth. Combining Eq. 3 with Eq. 4 yields the scan rate

$$
\frac{d f}{d t}=\left(\frac{1}{\mathrm{SNR}}\right)^{2}\left(\frac{P_{a \gamma \gamma}}{k_{B} T_{\mathrm{syst}}}\right)^{2} \frac{Q_{a}}{Q_{L}} \sim B^{4} V^{2} C^{2} Q_{L} T_{\mathrm{syst}}^{-2},
$$

which provides a measure of the experimental sensitivity for a given SNR.

\section{Experimental Implementation}

A conventional experimental setup and detection chain are illustrated in Fig. 1. The experimental setup consists of a cylindrical resonant cavity, embedded in a superconducting solenoid magnet, where axions interact with the virtual photons produced by the magnetic field and generate microwave photons via the inverse Primakoff effect. The power from the photon signal is magnified by multiple amplifications, down-converted to an intermediate frequency range, followed by the Fourier transformation. The axion signal appears as a peak in the power spectrum versus frequency. The rising point and spread of the energy peak correspond to the axion mass and kinetic energy, respectively.

The sensitivity of an experiment can be improved by enhancing the axion conversion power and signal-to-noise ratio. With fixed theoretical values, the axion-tophoton conversion power is enhanced by increasing the magnetic field, the volume of the cavity, the form factor of the resonant mode of interest and the quality factor of the cavity, as seen in Eq. 3. This is achievable by developing high field superconducting magnet and high $\mathrm{Q}$ cavity with a large volume and tuneablity for a large frequency range. Assuming these parameters are fixed, the signal-to-noise ratio can be improved by reducing the system noise temperature, as seen in Eq. 4. The dominant components of the system noise are the black body radiation from the cavity and the electrical noise from the first stage pre-amplifier. These noise sources can be reduced by placing the cavity at an ultra-low temperature and developing ultra-low-noise cryogenic amplifiers, such as superconducting quantum interference device (SQUID). For a given frequency scan range and limited scan time, the scan rate, Eq. 5, provides the best way to optimize the experimental sensitivity. 


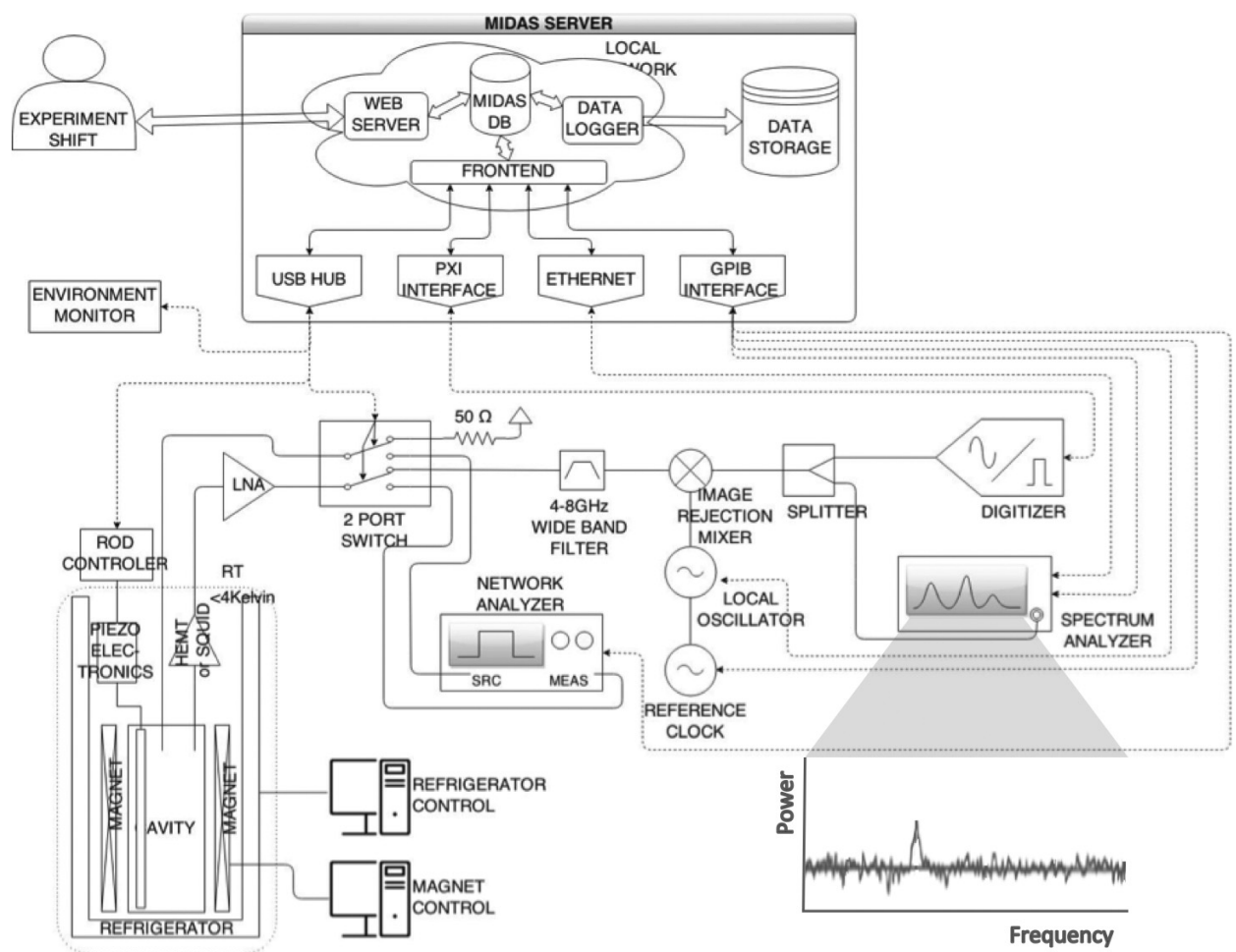

Fig. 1. A schematic illustration of experimental setup and receiver chain.

\section{Research Efforts at CAPP/IBS}

Research efforts carried out at the Center for Axion and Precision Physics Research $(\mathrm{CAPP})^{9}$ of the Institute for Basic Science (IBS) focus on improvements of the experimental parameters as described in the preceding section. The list of the major efforts is four-fold:

- development of a magnet providing a high field (most critical due to its fourth power),

- enlargement of the cavity volume within a limited magnet bore,

- design of a cavity with high Q,

- technology development for low system temperature.

These efforts are shown in Fig. 2.

\subsection{High magnetic field}

CAPP/IBS has launched a R\&D program with the magnet R\&D group of the Brookhaven National Laboratory (BNL) in New York, USA. This is a two-stage program consisting of $i$ ) development of a $25 \mathrm{~T}$ magnet with $10 \mathrm{~cm}$ of bore using 


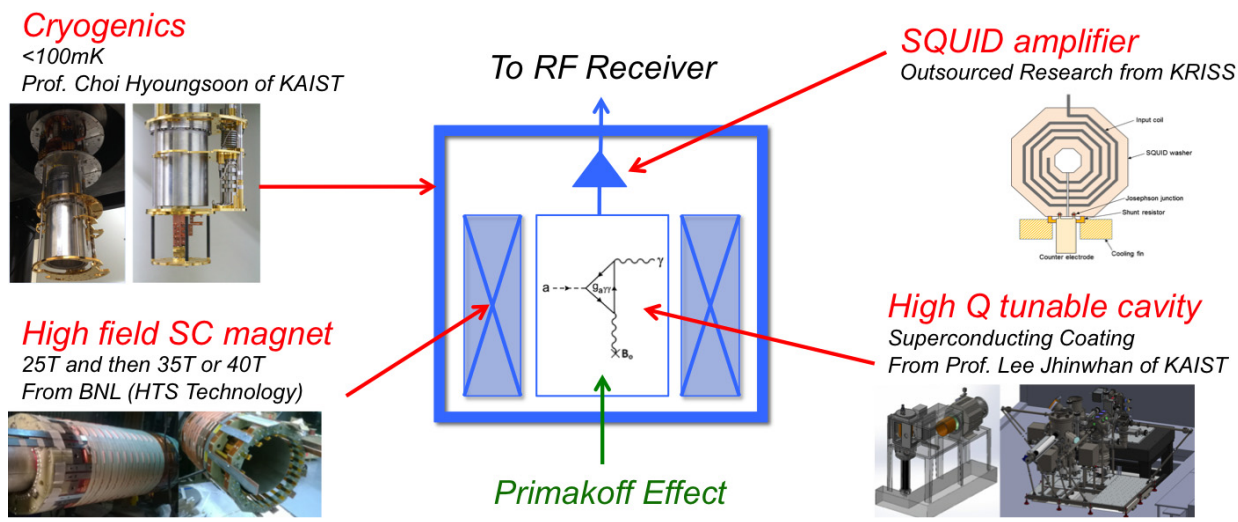

Fig. 2. Major R\&D efforts being conducted at CAPP/IBS in collaboration with several research institutes.

high-temperature superconductor (HTS) technology at the first stage; and ii) design of a hybrid magnet for $35 \sim 40 \mathrm{~T}$ by adding an outer layer of up to $15 \mathrm{~T}$ magnet, which is based on low-temperature superconducting technique, to the existing one ${ }^{\mathrm{a}}$.

\subsection{Large cavity volume}

Enlarging the magnet bore enables us to increase the cavity volume which thereby improves the axion signal power. On the other hand, exploring higher frequency regions requires a smaller size of the cavity as $\mathrm{TM}_{010}$ frequency scales inversely with the cavity radius, which in principle degrades the signal power. One of the most intuitive solutions to solve such a contradiction is to bundle small multiple cavities together and combine their individual outputs ensuring phase matching of the coherent axion signal. The first experimental result using a quadruple-cavity system was reported by ADMX in $2000 .{ }^{10}$ A calculation indicates that multiplecavity experiment is superiors to multiple single-cavity experiment in terms of SNR by a factor of $\sqrt{N}$, where $N$ is the cavity multiplicity. The challenging issue of this approach is phase-matching in both the frequency and time domains. The 5 -year Young Scientist program of IBS is dedicated to develop a phase-matching mechanism for multiple-cavity systems.

\subsection{High quality factor}

Improving the quality factor of cavities is one of the $\mathrm{R} \& \mathrm{D}$ efforts being performed at CAPP/IBS. The theoretical $\mathrm{Q}$ value of a typical copper cavity with annealing process is $Q \sim 10^{5}$, while that of the axions is $Q_{a} \sim 10^{6}$. In order to achieve higher $\mathrm{Q}$ value, a concept of a hybrid superconducting cavity has been considered by ADMX,

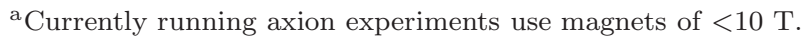


where the cavity wall is coated with thin-film of superconducting material while the end caps are made of copper. ${ }^{11}$ Expected improvement of the quality factor from this hybrid design is given by

$$
Q_{\text {hybrid }}=(1+L / R) Q_{\mathrm{Cu}}
$$

where $L$ and $R$ are the length and radius of the cavity, respectively. Our center performs a collaboration work with Prof. Lee Jhinwhan at the Korea Advanced Institute of Science and Technology (KAIST) for superconducting cavity development. The collaboration considers the design with all inner surfaces, including the end caps, coated with superconducting material, aiming at up to $Q \sim 10^{7}$ even under a high magnetic field. In the mean time, a comprehensive measurement of the residual resistance ratio ( $\mathrm{RRR})$ for high purity (>99.999\%) copper and aluminium in parallel as a part of the high-Q cavity developing project.

\subsection{Low system temperature}

In order to achieve an ultra low temperature, CAPP/IBS plans to employ dilution refrigerator (DR) technology. All DRs will keep the major component of the experiments, cavities, at as low as $10 \mathrm{mK}$ level, which will significantly reduce the physical temperature. The center plans to procure $7 \mathrm{DRs}$, consisting of four dry fridges and three wet fridges, at over several stages, each of which has its own purpose. Prof. Choi Hyoungsoon's group at KAIST and Dr. Chong Yonuk's group at the Korea Research Institute of Standards and Science (KRISS) are heavily involved in this project. Meanwhile, in order to develop low noise amplifier, the center has started a R\&D program with KRISS where Dr. Lee Yonho's group plays a leading role.

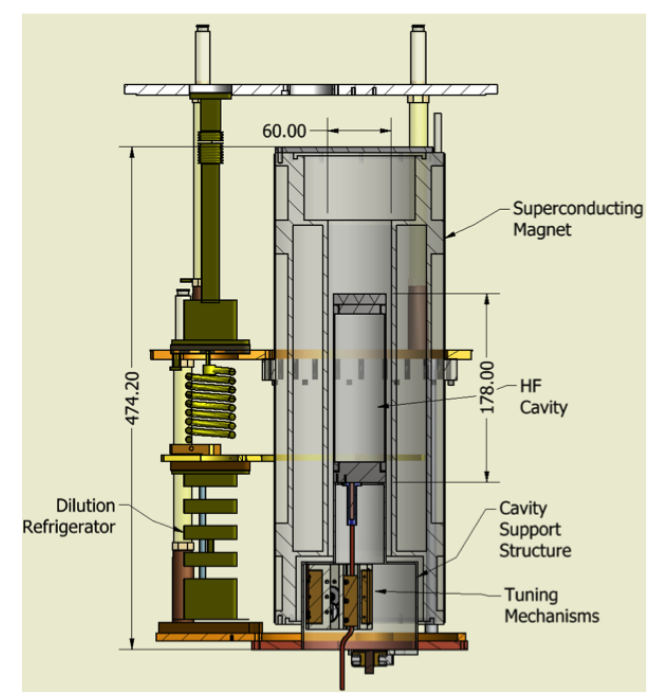

Fig. 3. Schematic view of the CULTASK experiment. The dilution refrigerator provides a capability of operating the cavity at $\sim 10 \mathrm{mK}$ and the superconducting magnet provides a $9 \mathrm{~T}$ field. 
The 5-year program will provide nearly quantum noise limited SQUID amplifiers to cover a broad frequency range (1 to $20 \mathrm{GHz}$ ), which will in turn deliver significant reduction of the system noise.

\subsection{CULTASK}

The CAPP Ultra Low Temperature Axion Search in Korea (CULTASK) is a corporate body of axion research efforts at CAPP/IBS, ${ }^{13}$ described in the preceding subsections. As of Oct. 2015, CULTASK is in engineering runs utilizing the dilution refrigerator with 9 T magnet at Prof. Choi's lab, as shown in Fig. 3. It has been building infrastructure including cavity design with reliable tuning mechanism, test of electric receivers at cryogenic and room temperatures, and receiver chain design.

\section{Projected Sensitivity}

Figure 4 shows the CAPP/IBS projected sensitivities for different scenarios depending on R\&D achievements at the center. A wide range of frequency (about $2 \sim 20$ $\mathrm{GHz}$, corresponding to about $8 \sim 80 \mu \mathrm{eV} / \mathrm{c}^{2}$ ) will be covered within next 5 years. The sensitivity from the experimental parameters currently available at the center is compatible with the expected one by the ADMX HF experiment. With a $25 \mathrm{~T}$ magnet, the experiment will be as sensitive as the KSVZ model, and additional

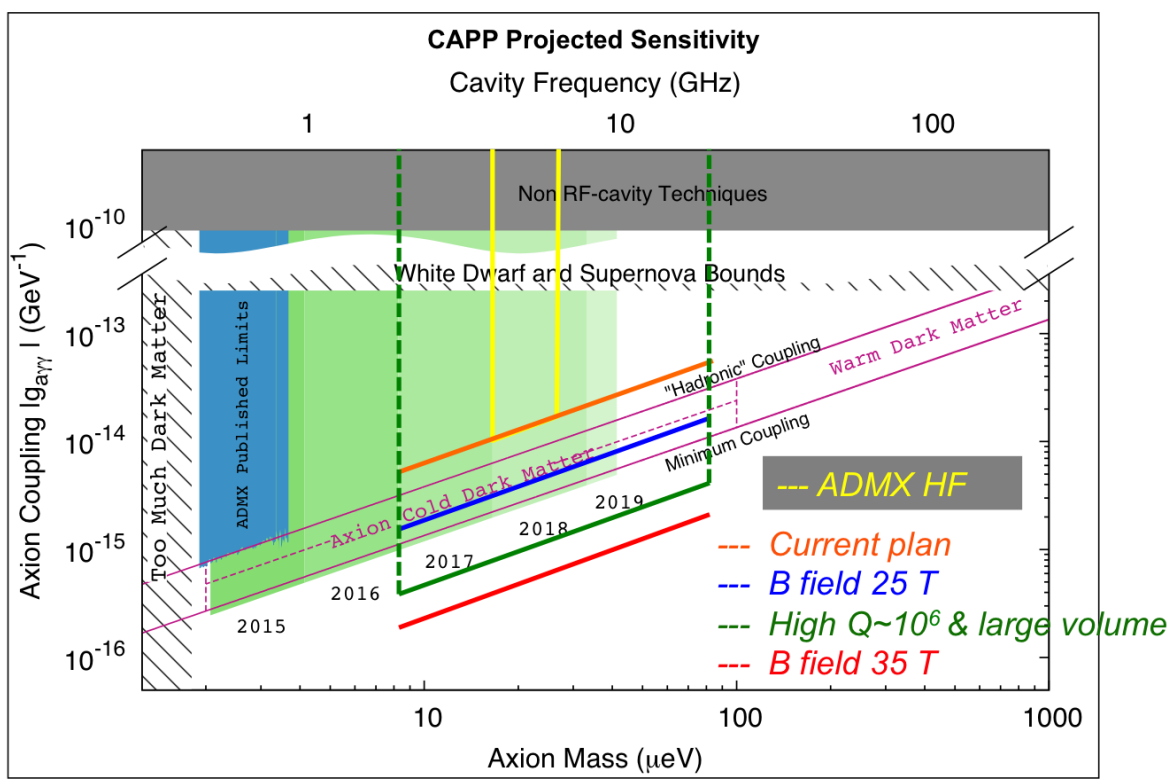

Fig. 4. Projected sensitivities by CAPP/IBS for the axion coupling versus its mass (frequency) for different scenarios represented by lines in different colors. The blue area corresponds to the published exclusion region by ADMX, ${ }^{12}$ while the green areas with different contrasts are the projected sensitivities by ADMX for the next 5 years. The yellow lines represent the limits expected by the ADMX-HF. 
improvements in the quality factor and volume of cavities will bring the sensitivity further down to the DSVZ model. Another interesting thing is that a higher magnetic field $(35 \mathrm{~T})$ will enable us to be sensitive to a scenario where axions compose $\sim 10 \%$ of the dark matter.

\section{Summary}

The axion is a promising particle not only to solve the strong CP-problem but also to address the dark matter issue. Cavity-based search is a promising approach to detect the faint axion-photon coupling. CAPP/IBS is a research center dedicated to axion dark matter searches in Korea. A variety of R\&D activities is underway at the center to improve the experimental sensitivity, expecting exciting results in a near future.

\section{Acknowledgments}

This work was supported by IBS-R017-D1-2015-a00/ IBS-R017-Y1-2015-a00. Special thanks go to my colleagues at CAPP/IBS.

\section{References}

1. N. Cabibbo, Phys. Rev. Lett. 10, 531 (1963); M. Kobayashi and T. Maskawa, Prog. Theor. Phys. 49, 652 (1973).

2. C.A. Baker et al., Phys. Rev. Lett. 97, 131801 (2006).

3. R.D. Peccei and H. Quinn, Phys. Rev. Lett. 38, 1440 (1977), Phys. Rev. D 16, 1791 (1977).

4. J. Preskill, M.B. Wise, and F. Wilczek, Phys. Lett. B 120, 127 (1983); L.F. Abbott and P. Sikivie, Phys. Lett. B 120, 133 (1983); M. Dine and W. Fischler, Phys. Lett. $B$ 120, 137 (1983).

5. A. Browman et al., Phys. Rev. Lett. 33, 1400 (1974).

6. P. Sikivie, Phys. Rev. Lett. 51, 1415 (1983).

7. J.E. Kim, Phys. Rev. Lett. 43, 2 (1979); M. Shifman, A. Vainshtein and V. Zakharov, Nucl. Phys. B 166, 493 (1980).

8. M. Dine, W. Fischler and M, Srednicki, Phys. Lett. B 104, 199 (1981); A. Zhitnitsky, Siv. J. Nucl. Phys. 31, 260 (1980).

9. Center for Axion and Precision Physics research (CAPP), Daejeon 34141 Republic of Korea. More information is available at http://capp.ibs.re.kr/html/capp en/.

10. D. S. Kinion, 2001, Ph.D. thesis (University of California, Davis).

11. K. V. Bibber and G. Carosi, arXiv:1304.7803 (2013) physics.ins-det, Status of the ADMX and ADMX-HF experiments; I. P. Stern, arXiv:1403.5332 (2014) physics.insdet, on behalf of ADMX and ADMX-HF collaborations, Axion Dark Matter Searches.

12. C. Hamann et al., Phys. Rev. Lett. 80, 2043 (1998); S. J. Asztalos et al., Astrophys. J. 571, L27 (2002); S. J. Aszgalos et al., Phys. Rev. Lett. 104, 041301 (2010); J. Hoskins et al., Phys. Rev. D 84, 121302(R) (2011).

13. W. Chung, The Coldest Axion Experiment at CAPP/IBS/KAIST in Korea, in Proc. 11th Patras Workshop on Axions, WIMPs and WISPs, eds. I.G. Irastorza et al. (Zaragoza, Spain, 2015), p.116 119. 